\title{
Study on ultrasonic testing of imperfect defects in concrete
}

\author{
Yuxuan Zhu, Shuqi Zhao, Ren Liu, Xingcheng Wang, Jipeng Kan, \\ Jinshuai Zhang, Meijing Wu, Weiyang Jiang, Dong Xiang*
}

School of Material Science and Technology, Shandong Jianzhu University, Jianan 250101, P. R. China

*Corresponding author. E-mail: xiang@sdjzu.edu.cn (Dong Xiang)

\begin{abstract}
Keywords: concrete; ultrasonic testing; imperfect; the plane for measuring method; defect Abstract. When there are imperfect defects in the concrete elements, it will have seriously impacted on the bearing capacity, durability and lifetime of the structure. Ultrasonic technology was used to effectively test the imperfect defect in concrete. The results show that the technology of the plane for measuring method can be adopted to detect the compactness of concrete. If the size of imperfect defects is smaller than the diameter of the probe, the detection errors should be larger, even be not tested. And it is more suitable for the larger size of imperfect especially more than $50 \mathrm{~mm}$. At the same time, the imperfect areas in concrete frequently exist in the corner position, and compactness in central location is good.
\end{abstract}

\section{Introduction}

Concrete is made up of cementitious material, granular aggregate (also known as aggregate), water, additive agents and admixtures added at certain times, which prepared according to certain proportion. Concrete materials, which have the characteristics of high compressive strength, good durability, wide strength range, rich raw materials and low price, are one of the most important civil engineering materials in the contemporary. They are widely used in shipbuilding, machinery industry, marine development, geothermal engineering and other industries [1]. However, the defects, such as pitted surface, cracks on concrete surface or cavities, imperfect etc., are formed in the process of mixing, stirring, molding and curing, due to the leakage of vibration, vibration insufficient, template slurry leakage or environmental conditions and other reasons. The internal imperfect defects will seriously effect on the bearing capacity of the structure and durability [2].

Nondestructive testing, with the help of an advanced instrument, equipment and technology, is the technical means for testing and analysis of the surface and internal defects under the premise condition of the detected object without damage [2]. At present, the nondestructive testing technology of concrete defects includes (1) the mechanical wave method-ultrasonic pulse wave, impact pulse wave, acoustic emission and so on; (2) the penetrating radiation method of ray and neutron flow [3]. For non-homogeneous concrete, the penetration ability of the ray is limited. The strength and internal defects of concrete are determined by basing on the change of ultrasonic propagation speed, amplitude, acoustic time and so on [4,5]. In 1949, Leslie successfully detected concrete by use of ultrasonic pulse. But, due to the low sensitivity, poor resolution and other restrict reasons of the instrument, and the fuzzy influence factors of ultrasonic testing for concrete, the engineering application of actual measurement was limited [6]. With the development of electronic technology and the continuous improvement of ultrasonic testing technology, the detection rate of cracks, imperfect or cavities was increased, and the variation law of caused strength was found out. Thus, the quality and service life of concrete were greatly improved [7 9]. When the ultrasonic tests the concrete, there are many advantages, such as the reliable results, simple operating process, strong penetration ability and convenient on-site testing etc.. Especially great advantages have showed in the testing of early strength of concrete, thickness, compactness and depth of crack etc.. However, 
because of the irregular and invisible of internal structure of concrete, ultrasonic testing of the defects is difficult to accurately quantify in concrete [10 11].

According to the structural characteristics and working conditions of engineering concrete, the test specimens of concrete were produced and selected. The positioning and quantitative analysis of imperfect defects in concrete were carried out by the plane for measuring method.

\section{Experiments}

Specimen preparation. Mix proportion of the testing concrete was 1:1.44:4.10:0.49 of cement:send:stone:water.The specimens, which size was $500 \mathrm{~mm} \times 300 \mathrm{~mm} \times 250 \mathrm{~mm}$, were made by using mechanical mixing, vibration, and natural curing indoor. Their age was $7 \mathrm{~d}$.

Method and equipment. In the experiment of concrete defect testing, many methods could be used, such as the plane for measuring method, the plane for slanting test method and drilling method etc.. $[10,12]$ When the non-metal ultrasonic detector (ZBL-U520) manufactured by Beijing ZBL (Zhi Bo Lian) Science \& Technology Co., Ltd. in this experiment was used, the plane for measuring method of ultrasonic pulse technology adopted to test the imperfect defect was convenient application and high precision.

Calculation and processing of acoustic parameters. According to CECS 21:2000 [4], the mean value and standard deviation of the sound value (or sound velocity), amplitude and frequency of concrete in a sample or a testing area are calculated by the Eq. (1) and (2).

$$
\begin{aligned}
& m_{x}=\frac{1}{n} \sum X_{i} \\
& s_{x}=\sqrt{\left(\sum_{i=1}^{n} X_{i}^{2}-n \cdot m_{x}^{2}\right) /(n-1)}
\end{aligned}
$$

Where $X_{i}$ is the measurement value of acoustic parameters (the sound value or sound velocity) at $i$-point; $n$ is measuring points of participation statistics.

The sound velocity or amplitude calculated by the sound value of each measuring point at a certain area are arranged in order from big to small, behind the obvious smaller data will be treated as a suspicious value. Then, the $m_{x}$ and $s_{x}$ value can be worked out by the biggest one in the suspicious data along with its front data according to Eq. (1) and (2), and the judgment value $X_{0}$ of abnormal situation can be calculated according to the Eq. (3).

$$
X_{0}=m_{x}-\lambda \cdot s_{x}
$$

If $X_{n} \geq X_{0}$, the $X_{n}$ and its subsequent data are considered as the abnormal values. When the $X_{n}$ is removed, $X_{1} \sim X_{\mathrm{n}-1}$ is calculated and distinguished until the abnormal values cannot be judged. Finally, the location range of the imperfect area in the concrete is determined according to the distribution of abnormal measuring points and waveform condition.

\section{Results and analysis}

The location and size of the imperfect defects in concrete were detected by the plane for measuring method, as shown in Fig. 1. The observed acoustic parameters were measured by the instrument, and the critical value of each acoustic parameter was calculated by the analysis software, which was named as the critical value 1 . The 20 measuring points of the acoustic parameters were tested by the specimen were shown in Table 1.

Comparing the acoustic parameter values of each point with the critical value 1 , the defect points can be obtained. The tested points around the defect points will be determined whether there are the existing abnormal positions. The critical value 2 is automatically calculated by the system, shown in Table 2. The final location of the defects is determined by the comparison values of two critical values. 


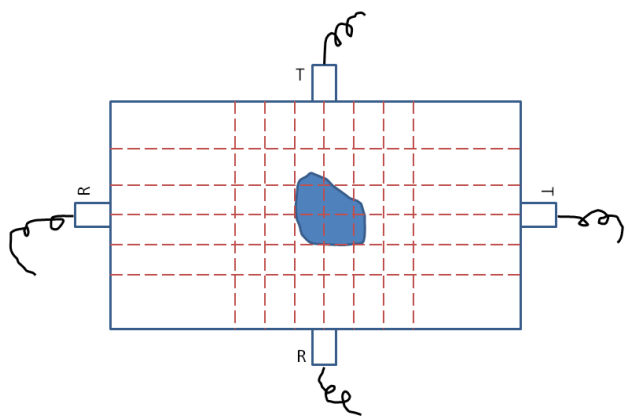

Fig. 1 The location and size of the imperfects tested by the plane for measuring method

Table 1 Acoustic parameters of the tested specimen

\begin{tabular}{ccccc}
\hline $\begin{array}{c}\text { Serial number } \\
\text { of tested points }\end{array}$ & $\begin{array}{c}\text { Tested distance } \\
(\mathrm{mm})\end{array}$ & $\begin{array}{c}\text { Acoustic time } \\
(\mu \mathrm{s})\end{array}$ & $\begin{array}{c}\text { Sound velocity } \\
\left(\mathrm{km} \cdot \mathrm{s}^{-1}\right)\end{array}$ & $\begin{array}{c}\text { Amplitude } \\
(\mathrm{dB})\end{array}$ \\
\hline $01-1$ & 400 & 95.20 & 4.202 & 96.85 \\
$01-2$ & 400 & 94.40 & 4.237 & 96.56 \\
$01-3$ & 400 & 96.80 & 4.132 & 92.42 \\
$01-4$ & 400 & 96.00 & 4.167 & 93.35 \\
$01-5$ & 400 & 96.80 & 4.132 & 91.96 \\
$02-1$ & 400 & 95.20 & 4.202 & 94.38 \\
$02-2$ & 400 & 97.20 & 4.115 & 95.91 \\
$02-3$ & 400 & 97.20 & 4.115 & 100.91 \\
$02-4$ & 400 & 98.40 & 4.065 & 100.81 \\
$02-5$ & 400 & 98.80 & 4.049 & 94.01 \\
$03-1$ & 400 & 94.80 & 4.219 & 96.33 \\
$03-2$ & 400 & 96.40 & 4.149 & 101.40 \\
$03-3$ & 400 & 98.80 & 4.049 & 101.01 \\
$03-4$ & 400 & 97.60 & 4.098 & 99.88 \\
$03-5$ & 400 & 98.00 & 4.082 & 97.20 \\
$04-1$ & 400 & 93.20 & 4.292 & 94.20 \\
$04-2$ & 400 & 97.20 & 4.115 & 97.90 \\
$04-3$ & 400 & 98.00 & 4.082 & 99.44 \\
$04-4$ & 400 & 97.20 & 4.115 & 98.45 \\
$04-5$ & 400 & 95.60 & 4.184 & 91.45 \\
$05-1$ & 400 & 96.40 & 4.149 & 87.74 \\
$05-2$ & 400 & 98.80 & 4.049 & 93.17 \\
$05-3$ & 400 & 100.00 & 4.000 & 90.19 \\
$05-4$ & 400 & 98.00 & 4.082 & 88.38 \\
$05-5$ & 400 & 95.60 & 4.184 & 83.05 \\
\hline
\end{tabular}


Table 2 The critical value 2 of the acoustic parameters automatically calculated by system

\begin{tabular}{ll}
\hline \multicolumn{1}{c}{ Items } & Results \\
\hline Average value of wave velocity $\left(\mathrm{km} \cdot \mathrm{s}^{-1}\right)$ & 4.136 \\
Standard deviation of wave velocity $\left(\mathrm{km} \cdot \mathrm{s}^{-1}\right)$ & 0.0646 \\
Critical value 1of wave velocity $\left(\mathrm{km} \cdot \mathrm{s}^{-1}\right)$ & 4.024 \\
Critical value 2of wave velocity $\left(\mathrm{km} \cdot \mathrm{s}^{-1}\right)$ & 4.053 \\
Average value of amplitude $(\mathrm{dB})$ & 96.55 \\
Standard deviation of amplitude $(\mathrm{dB})$ & 3.232 \\
Critical value 1of amplitude $(\mathrm{dB})$ & 91.16 \\
Critical value 2of amplitude $(\mathrm{dB})$ & 92.48
\end{tabular}

The numerical values of the tested points are lower than that of the critical points. These values are the abnormal points. It can be seen from Table 2 that there are 6 anomalie points of amplitude and sound velocity detected in the specimen, namely 6 defect location( 04-5, 05-1, 05-2, 05-3, 05-4, 05-5).

From Fig. 2, we could observe the location distribution of the defect points in specimen. There are 2 regional of the accumulation area of the abnormal points. Through the analysis of the acoustic parameters and waveform state of the abnormal points, the two regions are judged as imperfect. The cross positions, which are the horizontal line 5 and the vertical line 1,2,3 and 4, are recorded as "imperfect area 1"; and the cross locations of the horizontal line 4, 5 and the vertical line 5 are recorded as "imperfect area 2". It can be estimated that the range of imperfect area 1 is about 200 $\mathrm{mm} \times 50 \mathrm{~mm}$ and the range of imperfect area 2 is about $50 \mathrm{~mm} \times 100 \mathrm{~mm}$. Because the shape of imperfect area is irregular, two areas can be considered as a big imperfect area with the distribution location of the defect points in Fig. 2. The region is located at a lower part of the test specimen, which is about $50 \mathrm{~mm}$ around the horizontal line 5. It indicates that the compactness of the specimen at a lower edge part is worse, and the compactness of the upper and middle part is well.

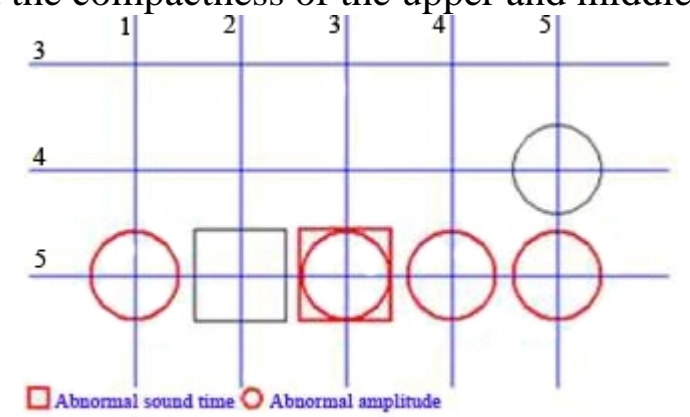

Fig. 2 The defect location of the specimen

\section{Conclusions}

Ultrasonic testing technology was used to detect the imperfect defects of concrete specimens. Through the calculation, comparison and analysis of test data, we can see that the detection rate and accuracy of the plane for measuring method are very high when the defect size is larger than the probe diameter or $50 \mathrm{~mm}$ length,. It can be seen in the test that the imperfect area of concrete is mainly distributed in the edge position of the specimen, while the heart part is often compactness. Ultrasonic testing can not only determine the location of imperfect defects in the specimen, and can measure the size range of this kind of defect, and then complete the process of the localization and quantification for ensuring the quality of concrete.

\section{References}

[1] Z. Q. Zhu, B. Yu, S. W. Mi, T. Yu and Y. Zhou: J. Cent. South Univ. (Science and Technology) Vol. 45(2014), p.3900 
[2] M. G. Yin and H. X. Liu: J. Yancheng Inst. Tech. (Natural Science Edition Vol. 20(2007), p. 75

[3] L. Han, C. Sun and Y. Wang: Urban Road Bridge and Flood Control Vol. 7(2011), p. 278

[4] CECS 21: 2000. Beijing: China Planning Press, 2000

[5] S. B. Li and H. X. Yang: Electronic Test Vol. 7(2013), p.252

[6] S. J. Popovics: J. Mater. Civil Eng. Vol.15(2003) , p. 211

[7] Y. H. Liu and X. B. Gao. Jilin Water Conservancy Vol. 5(2011), p. 27

[8] Y. H. Yang, T. Wang and Y. H. Wei: China Building Materials Science and Technology Vol. 5(2013), p. 10

[9] Y. Li, X. M. Liu and D. Z. Pan: J. High Way and Transportion Research and Development Vol. 21(2004), p. 83

[10] G. H. Dong: J. Yellow River Conservancy Institute Vol. 26(2014), p. 24

[11] Z. Liao: Science Technology and Engineering Vol. 11(2011), p.3114 